\title{
A high sensitivity refractometer based upon a long period grating Mach-Zehnder interferometer
}

\author{
T. Allsop, ${ }^{\text {a) }}$ R. Reeves, D. J. Webb, and I. Bennion \\ Photonics Research Group, Aston University, Aston Triangle, Birmingham B4 7ET, United Kingdom \\ R. Neal \\ School of Electronic Communications and Electrical Engineering, Faulty of Technology, University \\ of Plymouth, Plymouth PL4 8AA, United Kingdom
}

(Received 6 November 2001; accepted for publication 11 January 2002)

\begin{abstract}
A low cost interrogation scheme is demonstrated for a refractometer based on an in-line fiber long period grating (LPG) Mach-Zehnder interferometer. Using this interrogation scheme the minimum detectable change in refractive index of $\Delta n \sim 1.8 \times 10^{-6}$ is obtained, which is the highest resolution achieved using a fiber LPG device, and is comparable to precision techniques used in the industry including high performance liquid chromatography and ultraviolet spectroscopy. (C) 2002 American Institute of Physics. [DOI: 10.1063/1.1459093]
\end{abstract}

\section{INTRODUCTION}

The long period grating (LPG) is an ultraviolet (UV) light induced periodic modulation of the refractive index of the core of a single mode optical fiber with a typical modulation depth of $\sim 10^{-4}$ and a period in the range 100-600 $\mu \mathrm{m}$. The effect of the LPG is to introduce a number of attenuation bands into the transmissivity of the fiber, occurring at wavelengths where the LPG induces phase matched coupling between the guided and cladding modes. ${ }^{1}$ The study of the LPG attenuation bands has yielded many potential applications in telecommunications ${ }^{2}$ and the device has a number of possibilities in the field of sensing, since the mean wavelengths of the attenuation bands are sensitive to strain, temperature, and the refractive index of the surrounding medium. $^{3-7}$

In particular there has been strong interest in using LPGs as chemical sensors ${ }^{5,7}$ for the detection of, for example, organic aromatic compounds in paraffin. ${ }^{7}$ The sensitivity of the LPG arises from the dependence of the effective refractive index of a cladding on the refractive index of the surrounding medium, ${ }^{7}$ which can be observed through the spectral shift of its associated attenuation band. The conventional detection method is to measure these wavelength shifts using an optical spectrum analyzer. While this detection method is useful in the laboratory, it is not practical for industrial applications because of the size and cost of this instrument.

This article presents an attractive alternative scheme for refractive index sensing using an in-line fiber Mach-Zehnder interferometer based upon a pair of LPGs, forming the sensing element, and a heterodyne detection technique.

It has been shown that a pair of LPGs written in series along a fiber generates a series of interference fringes in each stop band of the transmission spectrum. ${ }^{8,9}$ These are caused by interference between light passing directly through the core and light that has been coupled to a cladding mode by

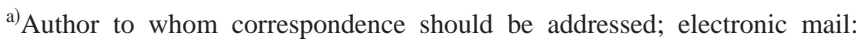
t.d.p.allsop@aston.ac.uk
}

the first LPG and then back into the core by the second. A theoretical treatment of these devices has been reported, ${ }^{9}$ and this has been subsequently refined to take into account the cladding mode effective index and group refractive index dependencies on wavelength. ${ }^{10}$

\section{ANALYSIS OF SENSING ELEMENT}

An LPG couples light from the core of the optical fiber into the modes of the cladding, generating resonance bands (attenuation bands) centered at wavelength $\lambda_{i}$ in the transmission spectrum of the guided core mode of the fiber. These cladding modes may decay rapidly due to scattering and bending. The center wavelength $\lambda_{i}$ of an attenuation band of a LPG with period $\Lambda$ is given by the phase-matching condition:

$$
\begin{aligned}
{\left[n_{\text {eff }_{\text {core }}}^{01}\left(\lambda_{i}, n_{1}, n_{2}\right)-n_{\text {eff }_{\text {cladding }}}^{1 \nu}\left(\lambda_{i}, n_{1}, n_{2}, n_{s}\right)\right] \Lambda } & \equiv \delta n_{\text {eff }} \Lambda \\
& =\lambda_{i},
\end{aligned}
$$

where $n_{\text {eff }}^{01}$ is the effective index of the core mode and $n_{\text {eff }}^{1 \nu}$ cladding is the effective index of the $\nu$ th cladding mode, both being dependent on the core refractive index $n_{1}$, the cladding refractive index $n_{2}$, and the wavelength $\lambda_{i}$. Impor-

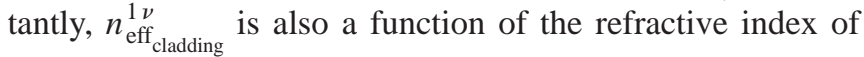
the surrounding medium $n_{s}$.

Our theoretical treatment of the LPG is based upon the analysis of a three-layered optical fiber waveguide. ${ }^{11,12}$ This analysis yields a dispersion relationship for the optical fiber, which is solved numerically.

\section{ANALYSIS OF DETECTION SCHEME}

There are a number of possible signal processing schemes that can be used to interrogate fiber interferometers. ${ }^{13}$ We have chosen to implement a phase generated carrier technique, ${ }^{14}$ as it permits interrogation of a passive sensing cavity, which is simpler (and, hence, poten- 
tially lower cost) than many alternative approaches. Also this technique can cope with the variable free spectral range encountered in LPG Mach-Zehnder interferometers and, in our application, can provide unambiguous measurement over many interference fringes. In this approach a small frequency modulation is applied to a laser illuminating the interferometer and the frequency modulation is then converted to a phase modulation by the finite path difference within the interferometer. Synchronous detection is performed on the output signal from the interferometric sensor, and the amplitudes of the components at the fundamental modulation frequency and its second harmonic are extracted. These two outputs represent the in-phase and quadrature components of the interferometer phase shift, ${ }^{15,16}$ and for small modulations these harmonics can be considered to give the first and second derivatives of the transmission profile of the sensing element. ${ }^{17}$

Consider a LPG Mach-Zehnder interferometer illuminated by a laser that has a sinusoidal modulation in wavelength at an angular frequency $\omega_{0}$ induced by the modulation of drive current. Using synchronous detection at $\omega_{0}$ and $2 \omega_{0}$ on the output of the sensor, we can obtain the amplitudes of the harmonics generated. It can be shown ${ }^{17}$ that the ratio of these two harmonics yields:

$$
\frac{\mathrm{Amp}_{\omega_{0}}}{\mathrm{Amp}_{2 \omega_{0}}}=\frac{J_{1}(\Delta \theta)}{J_{2}(\Delta \theta)} \tan (\Delta \phi),
$$

with

$$
\Delta \theta=\frac{2 \pi n L}{c} \frac{\delta v}{\delta i} \Delta i
$$

being the phase modulation induced within the interferometer, where $\delta v / \delta i$ is the current-to-frequency conversion factor of the distributed feedback (DFB) laser and $\Delta i$ is the current modulation amplitude applied to the laser. $J_{1}(\Delta \theta)$ and $J_{2}(\Delta \theta)$ are Bessel functions of the first kind and $\Delta \phi$ is the measured-dependent phase difference within the interferometer, given by

$$
\Delta \phi=\frac{2 \pi L}{\lambda_{\text {laser }}} \cdot \frac{d \delta n_{\text {eff }}}{d n_{s}} \Delta n_{s},
$$

where

$$
\frac{d \delta n_{\mathrm{eff}}}{d n_{s}}=F\left(n_{s}\right)
$$

is obtained from previous work ${ }^{7}$ and can be calculated for a given host fiber and a particular cladding mode.

Combining expressions (2) and (3), an equation is obtained that links the total phase change measured from the fiber LPG Mach-Zehnder interferometer to the change in the refractive index of the surrounding medium:

$$
\Delta n_{s}=\frac{\lambda_{\text {laser }}}{2 \pi L F\left(n_{s}\right)} \tan ^{-1}\left[\frac{J_{2}(\Delta \theta)}{J_{1}(\Delta \theta)} \cdot \frac{\mathrm{Amp}_{\omega_{0}}}{\mathrm{Amp}_{2 \omega_{0}}}\right] .
$$

A theoretical plot is presented in Fig. 1, showing the relationship between accumulated phase change of the LPG Mach-Zehnder interferometer and the refractive index of the

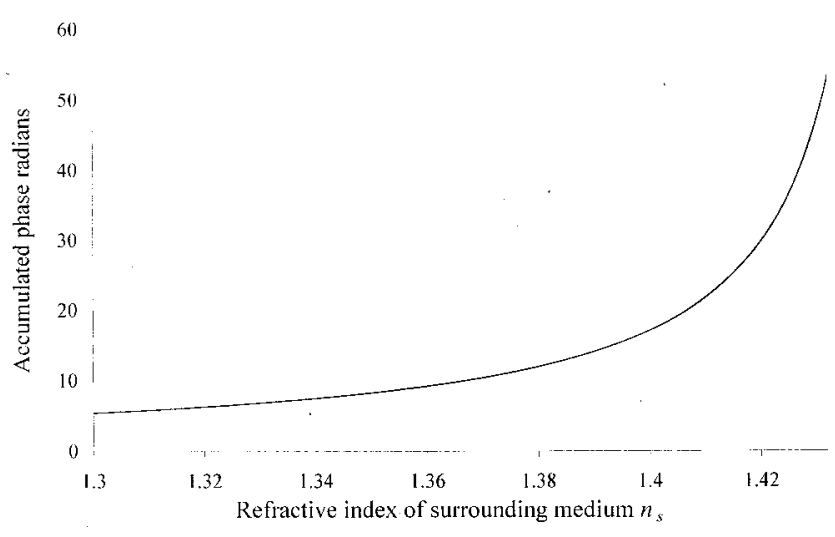

FIG. 1. The theoretical accumulated phase shift of the LPG Mach-Zehnder interferometer in the stop band of the 9th cladding mode in single mode B/Ge doped fiber.

surrounding medium $\left(n_{s}\right)$ for the 9 th cladding mode in a single mode $\mathrm{B} / \mathrm{Ge}$ codoped host fiber with an interaction length of $10 \mathrm{~cm}$ and a LPG separation of $20 \mathrm{~cm}$. The 9th cladding mode was chosen for two reasons. First the associated attenuation band of this mode matched spectrally with the wavelengths of DFB lasers available around $1550 \mathrm{~nm}$ and second, of the attenuation bands which did spectrally match the DFB lasers, this one was the most sensitive to changes in $n_{s}$. To generate Fig. 1, the effective index and group refractive index of the 9 th cladding mode as a function of $n_{s}$ for this fiber were determined from the method described above and substituted into Eq. (3) to obtain the interferometric phase. The functional form shown in Fig. 1 is to be expected since as $n_{s}$ increases more of the cladding mode extends into the surrounding medium leading to a supralinear increase in the effective cladding mode index. This effect also manifests itself as a chirp in the period of fringes in the stop band.

In Fig. 2 we show a parametric plot of the harmonics of the modulation frequency as a function of the surrounding index, assuming a modulation amplitude of $0.053 \mathrm{~nm}$. It may be seen that, as the index increases, a consequence of the reduction in the free spectral range of the interference fringes is that the amplitudes of the harmonics increases. As a result, the curve in Fig. 2 spirals outwards and a measurement of its

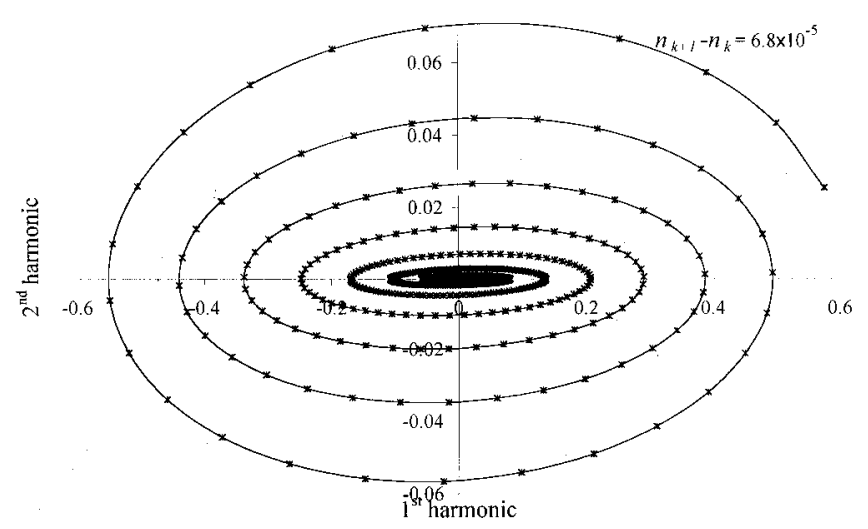

FIG. 2. Theoretical parametric plot of first and second harmonic of the modulation frequency, assuming a modulation amplitude of $0.053 \mathrm{~nm}$. 


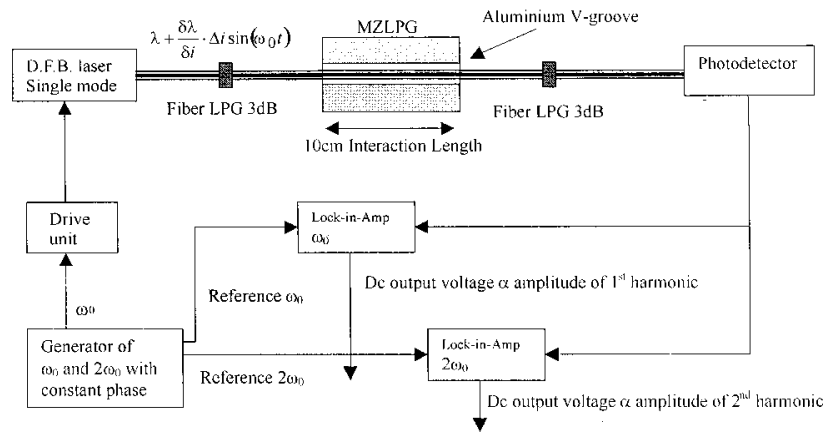

FIG. 3. Schematic of refractive index-sensing system.

radius can, in principle, be used to remove the $2 \pi$ phase ambiguity from the interferometer allowing unambiguous measurement of the external refractive index.

\section{EXPERIMENTAL PROCEDURE AND RESULTS}

An in-line fiber LPG Mach-Zehnder interferometer was formed by a pair of identical LPGs with strengths of $3 \mathrm{~dB}$ and a separation length of $20 \mathrm{~cm}$, which were fabricated in series along the core of a single mode optical fiber. The LPGs had a period of $240 \mu \mathrm{m}$ and were UV inscribed using a pulsed frequency quadrupled Nd:YAG (yttrium aluminum garnet) laser and an amplitude mask in a $\mathrm{B} / \mathrm{Ge}$ codoped fiber without $\mathrm{H}_{2}$ loading. This generated an attenuation band with a center wavelength at $1549 \mathrm{~nm}$ associated with the 9th cladding mode; the attenuation bands in this particular host fiber have been studied in previous work. ${ }^{7}$

The LPG Mach-Zehnder interferometer was placed between two supports and a $10 \mathrm{~cm}$ section (the interaction length) was located in a $\mathrm{V}$ groove. The $\mathrm{V}$ groove was made in an aluminum plate and machined to reduce bending of the fiber. The plate was placed on an optical table, which acted as a heat sink to maintain a constant temperature, thereby minimizing thermal fluctuations, which would induce spectral drift of the attenuation band or changes to the fringes within the attenuation band.

One end of the fiber was connected to a thermally stabilized pigtailed DFB laser, which illuminated the interferometer at a wavelength of $1549 \mathrm{~nm}$. The DFB laser was operated with a small sinusoidal wavelength modulation of amplitude of about $0.06 \mathrm{~nm}$ at a frequency of $5 \mathrm{kHz}$, which was obtained by modulating the injection current to the DFB laser. The opposite end of the interferometer was fed to a photodetector, the output of which was connected to two lock-in amplifiers, to perform synchronous detection. One of the lock-in amplifiers was referenced to a frequency of $5 \mathrm{kHz}$ and the other to $10 \mathrm{kHz}$, enabling measurement of the amplitudes of the first and second harmonics at the modulation frequency of the DFB laser, see Fig. 3.

The interaction length of the sensor was immersed in certified refractive index (CRI) liquids (supplied by Cargille Laboratories Inc.) which had a quoted accuracy of \pm 0.0002 . The changes in the amplitudes of the first and second harmonics were observed and measured when the interaction length was immersed in each of the CRI liquids. The parametric plot in Fig. 4 shows how the amplitudes of the two

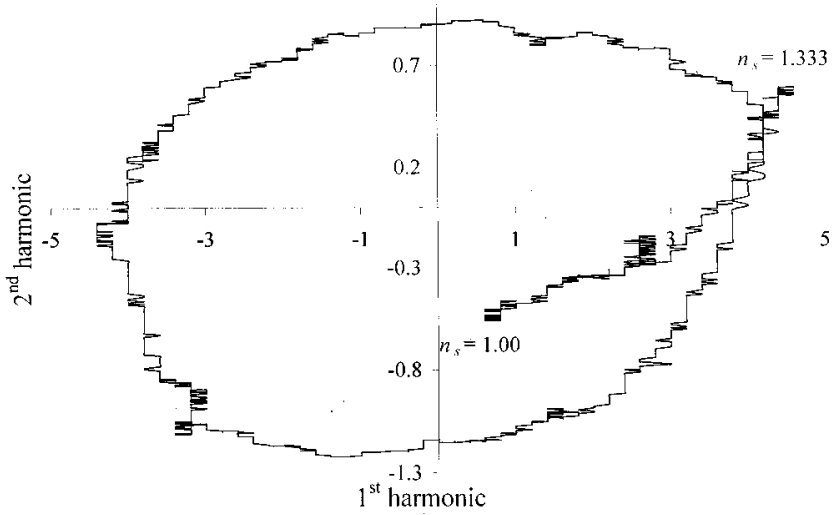

FIG. 4. Experimental plot generated from an in-line fiber LPG MachZehnder being submerged in deionized water.

harmonics changed as the interaction length, initially in air, was submerged in deionized water $\left(n_{s} \sim 1.333\right)$. The interaction length and $\mathrm{V}$ groove were carefully cleaned, washed in methanol, and then in deionized water and finally dried before the immersion into each CRI liquid, to reduce the quantities of salts being deposited on the fiber, thereby removing spurious refractive index changes.

After acquiring the amplitudes of the two harmonics from the series of CRI liquids, an inverse tangent was taken of the ratio of the first to second harmonics to generate the plot shown in Fig. 5.

The limiting error in this refractive index sensing scheme due to thermal drift was estimated from experimental data recorded over a period of $2 \mathrm{~h}$ to be $\Delta \phi_{\text {Total }} \sim$ $\pm 0.005 \mathrm{rad}$, during which time the room temperature fluctuated by less than $1^{\circ} \mathrm{C}$. Using data presented in Ref. 18, which relates to the temperature behavior of LPG attenuation bands written in this type of optical fiber, a figure of $\sim \pm 0.003 \mathrm{rad}$ was calculated assuming this level of temperature variation, in reasonable agreement with the experimental value. The experimental value is expected to be slightly higher as the theory does not take into account drifts due to the instrumentation. The stability of the sensing system in terms of the minimum detectable change in $n_{s}$ is dependent on the sensitivity of the cladding mode associated with the attenuation band to changes in $n_{s}$ and $T$ (spectral

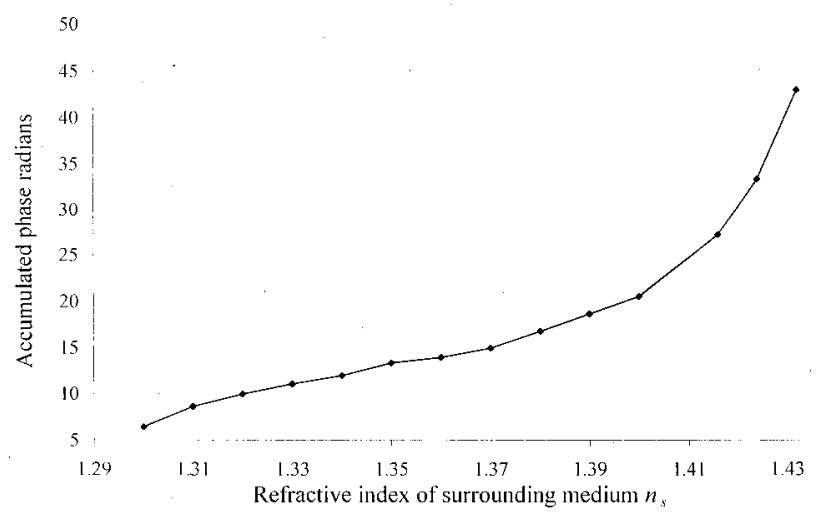

FIG. 5. The experimental accumulated phase shift detected using the fiber LPG Mach-Zehnder interferometer. 
TABLE I. The performance of the refractive, index sensing scheme using an in-line fiber LPG Mach-Zehnder interferometer.

\begin{tabular}{ccc}
\hline \hline$n_{s}$ refractive index & Phase stability & $\Delta n$ stability \\
\hline 1.33 & \pm 0.005 & $\pm 1.1 \times 10^{-5}$ \\
1.37 & \pm 0.005 & $\pm 4.0 \times 10^{-6}$ \\
1.43 & \pm 0.005 & $\pm 1.3 \times 10^{-6}$ \\
\hline \hline
\end{tabular}

sensitivity). ${ }^{7,19}$ Furthermore, as the value of the refractive index of the surrounding medium increases, so does the thermally induced wavelength shift of the attenuation band. ${ }^{7}$ A calculation of the measurement resolutions achieved at several values of index are given in Table I.

\section{DISCUSSION}

Comparing the experimental results obtained for this index sensing scheme against other index measuring systems, this approach appears quite attractive. First, Abbe refractometers have a resolution of $\sim 10-2 \times 10^{-5}$ (1.33 to 1.58 ) and are relatively expensive, whereas this system has better resolution and the potential for being low cost. Second, sensors have been demonstrated based on FBG evanescent field interactions ${ }^{20,21}$ that yield an index resolution of $\sim 10^{-3}$ (1.3) and $2 \times 10^{-5}(1.44-1.46)$ and have a more complex optical arrangement. Third LPG-based systems such as Refs. 7,21 , and 22 use an optical spectrum analyzer as part of interrogation technique yield index resolutions of $\sim 6$ $\times 10^{-5}$ (1.4) Ref. 7 and $\sim 3 \times 10^{-6}(1.438){ }^{21,22}$ This approach gives approximately a threefold increase in resolution without requiring an optical spectrum analyzer.

A refractive index sensing system has been demonstrated, which is based upon an in-line fiber long period grating Mach-Zehnder interferometer with a heterodyne interrogation technique. This sensing system has comparable accuracy to laboratory-based techniques used in industry such as high performance liquid chromatography and UV spectroscopy. The advantage of this system is that measurements can be made in situ for applications in continuous process control. Also, good agreement has been shown between the theoretical predictions for total accumulated phase change with respect to $n_{s}$ and the experimental results.

Compared to other refractive index sensing schemes using LPGs, this approach has two main advantages. First, the system has the potential for being low cost, requiring no optical spectrum analyzer. Second, so far as we are aware, it provides the highest refractive index resolution.

${ }^{1}$ S. Vasilev et al., Proc. SPIE 4083, 212 (2000).

${ }^{2}$ Y. Ruan et al., Proceedings of the SPIE Conference on Photorefractive Fiber and Crystal Devices: Materials, Optical Properties, and Applications V (SPIE, Denver, 1999), Vol. 3801, pp. 240.

${ }^{3}$ G. Meltz, W. Morey, and J. Dunphy, Proc. SPIE-Int. Soc. Opt. Eng. 1587, 350 (1992).

${ }^{4}$ V. Bhatia and A. M. Vengsarkar, Opt. Lett. 21, 692 (1996).

${ }^{5}$ A. M. Vengsarkar, P. J. Lemaire, J. B. Judkins, V. Bhatia, T. Erdogan, and J. E. Sipe, J. Lightwave Technol. 14, 58 (1996).

${ }^{6}$ H. J. Patrick, A. D. Kersey, and F. Bucholtz, J. Lightwave Technol. 16, 1606 (1998).

${ }^{7}$ T. Allsop et al., Opt. Commun. 191, 181 (2001).

${ }^{8}$ Yi Liu et al., Opt. Commun. 164, 27 (1999).

${ }^{9}$ E. Dianvo et al., Proceedings of 22nd ECOC, Oslo, MoB.3.6, 1996.

${ }^{10}$ B. Lee et al., Appl. Spectrosc. 38, 3450 (1999).

${ }^{11}$ T. Erdogan, J. Opt. Soc. Am. A 4, 1760 (1997).

${ }^{12}$ C. Tsao, Optical Fibre Waveguide Analysis (Oxford, New York, 1992).

${ }^{13} \mathrm{R}$. Willsch et al., Selected Papers on Fiber Optic Sensors (SPIE, Milestone Series, New York, 1995), Sec. 1.

${ }^{14}$ A. Dandridge et al., IEEE J. Quantum Electron. QE-18, 1647 (1982).

${ }^{15}$ K. A. Murphy et al., Opt. Lett. 16, 273 (1991).

${ }^{16}$ A. D. Kersey et al., Proc. Smart Struct. Mater. SPIE 1777, 61 (1992).

${ }^{17}$ T. Allsop et al., Meas. Sci. Technol. (submitted).

${ }^{18}$ F. Fifield and D. Kealy, Principles and Practice of Analytical Chemistry (S. Thrones, New York, 1995).

${ }^{19}$ X. Shu et al., IEEE Photonics Technol. Lett. 13, 818 (2001).

${ }^{20}$ K. Usbeck et al., Proc. SPIE 3483, 90 (1998).

${ }^{21}$ K. Schroeder et al., Meas. Sci. Technol. 12, 757 (2001).

${ }^{22}$ G. Laffont et al., Meas. Sci. Technol. 12, 764 (2001). 